\title{
Análisis sobre los procesos cognitivos implícitos en la comprensión de textos.
}

\author{
Sandra Acevedo Zapata ${ }^{1}$ \\ Lilia Cañón Flórez²
}

\begin{abstract}
Resumen
Esta investigación encuentra punto de partida en dos situaciones problemáticas fundamentales. En primera instancia, atiende a la creencia de que el estudiante al ingresar en la universidad, al haber superado niveles previos de escolaridad, se puede calificar de lector autónomo y, por tanto, se encuentra en capacidad de asumir de manera autónoma procesos de comprensión y de aprendizaje a partir de textos. En segundo lugar, reconoce la disyunción entre los diversos desarrollos teóricos alcanzados en relación con los procesos de comprensión de lectura y las prácticas adelantadas alrededor de estos procesos.
\end{abstract}

Ahora bien, cuando el ámbito universitario hace referencia a procesos de formación de las nuevas generaciones de maestros, indagar acerca de las estrategias cognitivas y metacognitivas en la comprensión de textos va más allá del reconocimiento de los propios procesos lectores y de las repercusiones en el actuar como estudiantes universitarios: da lugar a que, a partir de conocerse y reconocerse como lector, se abran caminos que recaerán en las futuras formas de encaminar actos de enseñanza.

Desde el punto de vista metodológico, el proyecto se centra en la construcción del estado del arte sobre los abordajes teóricos que se han hecho sobre la comprensión de textos académicos y los procesos cognitivos implícitos.

Palabras clave: comprensión de textos, textos académicos en educación superior, comprensión lectura, metacognición, procesos cognitivos.

1. Licenciada en Psicología y Pedagogía. Magister en Educación. Docente Asistente Universidad Nacional Abierta y a Distancia.sandra.acevedo@unad.edu.co. Grupo de investigación: cibercultura y territorio.

2 Maestría en Lingüística Española. Instituto Caro y Cuervo. Actualmente se desempeña como docente de la Universidad Pedagógica Nacional y Universidad de la Salle. 


\title{
Reflection about the cognitive processes underlying in text comprehension
}

\begin{abstract}
This research takes as a point of departure two fundamental problem situations. Firstly, it addresses the assumption that as they reach college and having passed previous schooling levels, students can be considered autonomous readers who thereby are skilled to undertake written text-based learning and comprehension processes. Secondly, it sees a disjunction between the various theoretical developments made on reading comprehension processes and practices developed around those processes.

However, when it comes to formation processes for new teacher promotions, inquiring about cognitive and metacognitive strategies in reading comprehension goes beyond recognizing reading processes themselves and their implications on university students behavior - it helps open new ways that will have an effect on the subsequent ways to lead learning acts, based on their own acknowledgment as readers.

From a methodology point of view, this project focuses building a state-of-the-art on theoretical approaches made on scholarly texts comprehension and underlying cognitive processes associated to them.
\end{abstract}

Keywords: text comprehension, academic writing in higher education, reading comprehension, metacognition, cognitive processes.

\section{Introducción}

En el ámbito universitario la lectura se presume esencial tanto para el aprendizaje como para dar respuesta a las exigencias del desempeño académico, en especial porque gran parte de los procesos académicos giran alrededor del texto escrito: la lectura se categoriza como un medio fundamental para el acceso al aprendizaje, la adquisición de información y el acercamiento a procesos de formación. Por eso, comprender un texto se convierte en tarea básica en el actuar académico. 
Los avances en los procesos de escolaridad exigen mayores niveles de lectura independiente y de comprensión lectora. En términos generales, se concibe que los estudiantes universitarios hayan pasado por procesos de lectura que les permite enfrentarse de manera adecuada a las exigencias y necesidades del ámbito universitario; se espera, entonces, que estén en capacidad de enfrentar por su cuenta textos académicos. Pero, de forma contraria, se ha encontrado que gran cantidad de estudiantes universitarios evidencian algunos problemas y dificultades en la comprensión de textos. Parece que el acercamiento a la comprensión literal se logra; pero cuando la complejidad del texto se hace mayor, los niveles de comprensión decaen.

El estudio en la comprensión de textos no es novedoso. En las últimas décadas se han alcanzado importantes aportes en el análisis de los procesos de lectura y de sus componentes; enfoques que han entregado aportes teóricos y prácticos. Nuevas herramientas y nuevos instrumentos de análisis y conceptos han contribuido a una mejor explicación del proceso, de cómo se representan los contenidos del texto y de qué estrategias entran en juego.

Sin embargo, ubicar elementos de relación entre los avances teóricos y su posible aplicación continúa en el orden del día. En el contexto de formación universitaria, urge acercarse y estudiar por qué en diversas ocasiones los escenarios de acción de estrategias de comprensión lectora no reflejan los avances teóricos planteados. Por ejemplo, todavía prevalecen las llamadas formas tradicionales que no alcanzan a abordar la complejidad de la problemática de la lectura y continúan centradas en la exclusiva mirada de habilidades dirigidas hacia comprensiones literales de los textos.

Desde esta perspectiva, se considera que antes de proponer vías de solución a partir de supuestos problemas en procesos de comprensión lectora para llegar a resultados específicos, se hace necesario reconocer, desde la concepción de la lectura como proceso interactivo y transaccional, no solo el déficit del lector frente al texto sino más bien cuáles son las formas cómo ese lector y los procesos académicos que lo acompañan generan estrategias de comprensión. En términos de medicina se diría que se está centrando en la enfermedad y no en el enfermo. En otras palabras, el problema no se ubica en la deficiencia; se plantea en los procesos, de ahí que se hace pertinente preguntarse ¿Cuáles son los hallazgos de las investigaciones sobre los procesos cognitivos implícitos en la comprensión lectora de textos académicos en educación superior?

De esta forma, con el desarrollo del presente trabajo, se buscó identificar, sistematizar los hallazgos de las investigaciones sobre los procesos implícitos en la comprensión lectora de textos académicos en educación superior. 


\section{Metodología}

Desde la perspectiva cualitativa se abordó la pregunta ¿Cuáles son los hallazgos de las investigaciones sobre los procesos de formación en la comprensión lectora de textos académicos en educación superior?, para ello se realizó una investigación en la cual se abordaron las siguientes fases:

Fase descriptiva: se realizó una descripción de las investigaciones sobre los procesos implícitos en la comprensión lectora de textos académicos en educación superior.

Meta 1. Delimitación del problema y categorías: diseño de instrumentos de recolección de información.

Meta 2. Rastreo documental con aplicación de formatos por categorías de investigaciones en procesos de comprensión de textos académicos en educación superior.

Fase de sistematización: la información recolectada en la fase descriptiva se sistematizó en matrices de doble entrada por categorías de análisis y actores del proceso de investigación.

Meta 3. Sistematizar investigaciones de procesos de formación en comprensión de textos en educación superior.

Meta 4. Diseñar y alimentar matriz de doble entrado con categorías y resultados de investigaciones y experiencias.

Fase analítica: con la información recolectada se realizó un análisis comparativo por investigaciones o experiencias y categorías de análisis sobre los procesos de comprensión de textos académicos en educación superior.

Meta 5. Analizar información por categorías y hallazgos para construir el estado del arte de las investigaciones y experiencias de procesos de formación en comprensión de textos en educación superior.

\section{Resultados y discusión}

\section{La lectura como proceso}

El proyecto se interesó por la comprensión de lectura en el ámbito académico universitario. 
De acuerdo con lo hasta ahora expuesto, vale la pena incorporar el concepto de leer para aprender de Beaugrande (1984). Concepción que implica reconocer el pensamiento reflexivo, analítico y crítico de un lector intencional, autónomo e independiente y, en consecuencia, agente activo y responsable de su propio aprendizaje.

Algunos teóricos toman el proceso de comprensión de lectura desde la perspectiva del texto; otros, desde la perspectiva del lector. En el presente estudio se parte de un proceso de comprensión centrado en el lector; sus esquemas de conocimiento previo, sus expectativas, sus preferencias y sus metas de lectura. Se justifica tal postura al considerar que del lector y de lo que aporta al proceso depende la construcción del significado del texto.

En concordancia, la comprensión de la lectura se reconoce como un proceso gradual y estratégico de creación de sentido, a partir de la interacción del lector con el texto en un contexto particular. Dicha interacción se encuentra mediada por el propósito de lectura, el conocimiento previo y las expectativas del lector; además, le permite involucrarse en procesos de inferencia necesarios para construir y revisar una representación de lo expuesto en el texto.

Como proceso, la comprensión se va construyendo desde cuando el lector se enfrenta al texto, desde cuando toma la decisión de leerlo y se formula expectativas en relación con sus contenidos. En la interacción lector-texto se activan esquemas cognitivos pertinentes del lector $\mathrm{y}$, a partir de la confrontación permanente de los esquemas con la información que va procesando, el lector se involucra de manera activa en un proceso de inferencias que le permitirá llevar a cabo de operaciones cognitivas en su intento de construir el significado del texto y de elaborar una representación coherente de sus contenidos (González-Marqués, 1991).

La activación, la revisión, la construcción y el uso adecuado de esquemas cognitivos, junto con la elaboración de inferencias se constituyen en actividades fundamentales a las cuales acude permanentemente el lector durante la lectura para el procesamiento y comprensión del texto. Ahora bien, el uso de tales esquemas ha de convertirse en una actividad predeterminada que, junto con los procesos de inferencia, hará posible interpretaciones más ajustadas a los requerimientos académicos de lectura autónoma.

Finalmente, en la medida que el texto en sí determina el significado sólo en parte, se reconoce la lectura como un proceso interactivo, intencional, constructivo e inferencial caracterizado por la formación y la comprobación permanentes de hipótesis acerca del contenido del texto (Smith, 1971; Goodman, 1967).

Proceso de comprensión de textos

El tema de la comprensión de la lectura ha sido siempre polémico, entre otros aspectos, por la ausencia de un modelo universalmente aceptado acerca del 
proceso lector. Pero no ha de negarse la importancia de la comprensión lectora. En el cierre del anterior siglo se dieron grandes y numerosos desarrollos en el área de la lectura y la comprensión de textos. En el aspecto de interés en el presente trabajo, se pudo concluir que la lectura se debe reconocer como un proceso cognitivo altamente complejo que involucra una serie de operaciones mentales o cognitivas. Este contexto permitió también establecer la categoría de lector competente para designar a quien puede moverse automática y rápidamente de los niveles inferiores a los superiores de negociación de significados; negociaciones a las cuales dedicará gradual atención y esfuerzo cognitivo (Dubin y Eskey: 1986).

Un recorrido histórico muestra cómo en la década de los años setenta los objetivos de un curso de lengua se definían en términos de las cuatro habilidades lingüísticas: hablar, escuchar, leer y escribir. Modelo según el cual hablar y escribir se constituyen en eventos productivos que demandan un sujeto activo; por su parte, escuchar y leer se consideran receptivos y configuran un sujeto pasivo. Una década después, se hizo evidente que ninguna de las habilidades de la lengua es pasiva.

En relación con el desarrollo teórico acerca de la lectura y de la comprensión de textos se ha de mencionar, sin duda, el panorama expuesto por J. Orasanu y M. Penney (1986). La lectura, en la visión tradicional, se llevaba paso a paso desde la percepción de letras para formar palabras y de su combinación hasta completar oraciones; se pasaba, automáticamente, a la comprensión. Se insistía en la práctica de la decodificación y se dedicaba poca atención a la comprensión y a los problemas o dificultades de lectura que se resolvían con más práctica en la decodificación. Después de años de investigación en distintas disciplinas como la sicología, la lingüística, la antropología, la pedagogía y el procesamiento de información, interesadas en el fenómeno de la lectura, el concepto de compresión empezó a reevaluarse.

Los estudios lingüísticos de los años sesenta, desde la sicolingüística, mostraron que las concepciones estructuralistas previas no explicaban ni la creatividad de la lengua ni la posibilidad de comprender enunciados nuevos (Miller, 1965, citado por Orasanu, 1986). Se llegó a establecer que el significado de las oraciones no es equivalente a la suma de los significados de las palabras y que la enseñanza a partir de la práctica de repetición como refuerzo no garantizaba la adquisición o el aprendizaje de una lengua. Se admitió, por ejemplo, que en la comprensión y en la producción del discurso el sujeto no sólo reconoce o produce oraciones aisladas, con significado local, sino que, además, reconoce su valor y función, o se los asigna, como parte de un todo coherente, del sentido global del texto (Widdowson, 1978).

En el área de la psicología cognitiva, los trabajos de Bruner caracterizaron la mente como una "máquina de inferencias" que interpreta y organiza lo nuevo 
a partir del conocimiento previo; así, se aleja de la conceptualización que ve la mente provista de un "input" que se acciona frente al manejo de información.

En la década de los años sesenta, la preocupación en el área del procesamiento de información se centraba en construir máquinas que pudieran "pensar" (Newell, 1980; Simon, 1992; Minsky, 1975; entre otros). En consecuencia, se desarrollaron modelos de los procesos cognitivos humanos y metáforas del procesamiento humano de la información procesos paralelos, secuenciales e interactivos del procesamiento de información, de la memoria de trabajo y de las formas de representación mental del conocimiento.

Aún cuando la lingüística del texto no reconoce como una teoría o método único, designa el trabajo que desde la lingüística se hace con el texto como objeto primordial de estudio (Van Dijk, 1979). Al nutrirse de la antropología, entiende la significación como una actividad humana y, en consecuencia, mira el texto como uno de los "artefactos culturales", (Malinowski, 1923, citado por de Beaugrande y Dressler, 1983). Se reconocieron las relaciones entre la lengua y situación de comunicación, entre los patrones de habla y los roles sociales. El interés en el texto como unidad de análisis surge como reacción a las teorías que ven la oración como la máxima unidad de significación y que no consideran la selección y el uso de las estructuras como el resultado de operaciones intencionales. Se pasa, de este modo, del análisis de las estructuras de la lengua al análisis de la construcción de esas estructuras.

La ciencia del texto ha formulado algunos de los supuestos que vale destacar: los textos son vehículos de interacción intencional, la organización de las ideas en el texto es objeto de control sistemático y consciente, la valoración de un texto depende de sus efectos sobre la audiencia (de Beaugrande y Dressler, 1983). Se empiezan a considerar, entonces, aspectos relacionados con la producción, la presentación y la recepción del texto. Desde el análisis del discurso, la ciencia del texto ha recibido algunos aportes. La consideración de los mecanismos como cohesión, coherencia, intencionalidad, aceptabilidad, situacionalidad, intertextualidad e informatividad que de la combinación de estructuras un aspecto relevante en una situación de comunicación particular (Sinclair y Coulthard, 1975; Coulthard, 1977). Los trabajos de Van Dijk $(1972,1977,1979)$ en relación con textos literarios y poéticos aportan la noción de "macroestructura" o idea principal que expresa el contenido del texto. Más adelante, Van Dijk acude a la sicología cognitiva para postular un modelo del texto como proceso.

En términos epistemológicos, el presente proyecto pone mayor énfasis en los procesos de construcción del significado del texto y, sin negar su importancia, asume la decodificación como un medio y no como un fín en sí mismo. La diferencia crucial entre esta visión y la "antigua" reside en la concepción de la lectura como un proceso de creación de significados por parte del lector; se ve 
como un proceso activo y flexible a partir del uso de estrategias que dependen de un propósito previo de lectura y del texto. Como expresa Widdowson (1984), la lectura no es, entonces, mera decodificación sino un acto comunicativo que involucra la interacción entre los esquemas del lector y el texto en la búsqueda y construcción de significados.

\section{Papel de los esquemas cognitivos en la comprensión de textos.}

Según la teoría de los esquemas, los conocimientos previos del lector se encuentran almacenados de manera organizada en la memoria a largo plazo y disponibles en esquemas. Rumelhart (1980) define esquema como el conjunto estructurado de conocimientos y de procedimiento de acción disponibles que se ejecutan en distintos ámbitos de acción específicos. Los esquemas se asemejan a teorías acerca de la naturaleza de eventos, objetos o situaciones que el ser humano enfrenta, pero se caracterizan por ser informales y privados.

El total de esquemas utilizados frente a una situación o un texto particular se constituye en modelo interno de esa situación en ese momento o de lo expuesto en ese texto en particular. Por tanto, el conjunto total de esquemas de que se dispone para interpretar el mundo sería, en cierto modo, una teoría privada de la naturaleza de la realidad (Rumelhart, 1984). Podría hablarse de un proceso cíclico en el uso de los esquemas, puesto que a partir de esa teoría privada de la realidad se llega a la interpretación de una situación o de un texto y, a la vez, esta interpretación se integra con la interpretación que del mundo en general tiene el lector. El proceso dado a partir de la interacción entre los esquemas y el mundo hace posible aumentar y refinar permanentemente el conocimiento (López, 1997).

La teoría de los esquemas señala que, cuando el lector se enfrenta a un texto, la confrontación de los contenidos con sus esquemas previos le permite crear ciertas expectativas en relación con el contenido del texto; sería un proceso ascendente. A medida que se avanza en la lectura, si la información del texto encaja en los esquemas previos se confirman las expectativas iniciales; entonces, el lector puede codificar la información nueva para integrarla a los esquemas existentes; este sería un proceso descendente. Si la información no encaja en los esquemas, puede ser que no haya codificación y, en consecuencia, no se podrá dar la comprensión del texto o se podría llegar a una distorsión de la información, puesto que el lector intenta acomodarla en sus esquemas previos. Sin embargo, en estas situaciones es posible también, y deseable, que el lector decida revisar de alguna manera sus esquemas existentes, los complete, amplíe o precise para poder interpretar de modo adecuado los contenidos del texto.

Parece ser que al enfrentarse al texto, algunas claves del mismo "disparan" esquemas asociados que el lector posee -proceso ascendente- y éstos a su vez, actúan como marco de referencia para la interpretación de los contenidos del texto -proceso descendente- (Spiro, 1979). Si el lector no posee los esquemas 
adecuados, no los activa o no los usa de manera apropiada, no logrará involucrarse en un acertado proceso de comprensión (una de las diferencias cruciales entre lectores eficientes y no eficientes).

Diversos autores (Spiro, 1979; Garner, 1987a) han encontrado que los lectores no eficientes tienden a centrar su atención en lo conocido y procesan superficialmente lo no conocido. Además, debido también a su falta de esquemas adecuados, no son flexibles en su manera de procesar el texto y tienden a confiar excesivamente en un proceso ascendente, a partir de los datos del texto, sin ajustar el proceso a sus necesidades y a un propósito de lectura previo.

Así pues, la comprensión como proceso de construcción de significados depende, en gran medida, de la existencia, de la accesibilidad y del uso apropiado de los esquemas. Éstos permiten el proceso y la comprensión del texto durante el proceso lector y, a la vez, se actualizan durante el mismo proceso. Permiten así al lector la adquisición, la revisión o la ampliación de conocimiento y lo llevan a crear nuevo conocimiento (Bransford, 1985; Tiernay y Pearson, 1985): una vez actualizado el conocimiento, se da la integración semántica y el conocimiento resultante se almacena de manera organizada en la memoria a largo plazo donde queda disponible para su uso en situaciones posteriores.

El texto y la comprensión de lectura. Como se ha señalado, el texto es más que la suma de las palabras o de las oraciones impresas en una página; consiste en un modo de expresión que cumple una función comunicativa en la interacción humana (Petoffi, 1979, citado por de Beaugrande y Dressler, 1983) y para que el texto cumpla dicha función debe reunir ciertos rasgos de textualidad.

Varios autores se han ocupado de estudiar los rasgos que le dan textura o textualidad al texto. Beaugrande y Dressler ven el texto como una ocurrencia comunicativa que cumple siete estándares de textualidad para que sea comunicativo (de Beaugrande y Dressler, 1983:3). Tales rasgos están definidos no por el texto en si, sino por éste y la situación de comunicación donde es producido y recibido; en consecuencia, se tienen algunos rasgos más directamente dependientes e internos al texto: la cohesión y la coherencia.

Otros se relacionan con la interacción comunicativa: los participantes y el contexto. En relación con los participantes: la intencionalidad, la aceptabilidad y la informatividad. En relación con el contexto:la situacionalidad y la intertextualidad. Otros autores coinciden en asignar al texto cuatro características básicas que le dan su carácter comunicativo (Alliende, 1982, Anderson y Armbruster, 1984, Singer, 1985, Vidal-Abarca, 1991): la estructura, la coherencia, la unidad y la adecuación.

De otra parte, los trabajos orientados al análisis de los distintos tipos de textos han mostrado que la manera como se organiza y estructura la información, 
las formas lingüísticas escogidas para expresar esa información y las marcas o señales retóricas o discursivas que se usen dependen de la intención comunicativa del autor y de la audiencia que éste tiene en mente. Al respecto, distintos autores plantean tipologías textuales, resultantes de diferentes criterios de clasificación; pero, en términos generales, la mayoría coinciden en clasificar los textos como narrativos, expositivos, descriptivos, instruccionales y argumentativos, aunque no se debe hablar de una tipología textual única.

El conocimiento de los textos, de la manera como se organizan, de sus marcas discursivas, conforma esquemas estructurales, formales o textuales; esquemas cognitivos, previos esenciales para la construcción del significado del texto. Aun cuando una parte de tales esquemas formales se adquiere por la experiencia con una diversidad de textos que circulan en el entorno, se espera que los docentes, en atención al trabajo interdisciplinario - base del actual modelo de educación superior aseguren, mediante un trabajo sistemático, estrategias de lectura de distintos tipos de textos académicos, trabajo que redundará en un mejor proceso de comprensión y aprendizaje.

\section{Metacognición y lectura.}

El concepto de metacognición se ha aplicado en distintos campos: memoria, atención, solución de problemas, estrategias, comprensión del lenguaje, aprendizaje. Su significado es aún bastante variable. Dos orientaciones han sido base fundamental en el área: de una parte, los trabajos que la psicología ha cognitiva ha realizado en cuanto a la metacognición, en gran mayoría con niños; de otra, los trabajos adelantados con adultos y que se relacionan con el proceso de información en cuanto al control ejecutivo (Fischer y Mandl, 1984; Garner 1987a).

De los hallazgos realizados, se destaca la necesidad de tener en cuenta las variables tanto metacognitivas como de control ejecutivo en el comportamiento del sujeto en tareas de procesamiento y comprensión de textos. Tener en cuenta estas variables permitirá: entender la relativa insuficiencia o eficacia de las estrategias de lectura y estudio; acercarse a los aspectos extratextuales propios del contexto que del texto mismo y relacionados más con el lector, con sus esquemas previos y con las estrategias que utiliza en función de la situación en particular; entender que ante la variedad de propósitos y de tareas se utiliza una variedad de actividades coordinadas y orientadas a tales propósitos y tareas; establecer que los conocimientos previos del lector y lo que aporta al proceso de lectura son fundamentales; y, finalmente, hacer evidente la necesidad de presentar y practicar un gran número de estrategias orientadas tanto al logro cognitivo en el proceso de lectura, como al control y supervisión del proceso.

Metacognición. La comprensión del texto, como proceso estratégico, supone que el lector, con base en su propósito de lectura, siga un plan lo suficientemente flexible que se pueda ir ajustando al propósito, al tipo de texto, a las demandas 
de la tarea y a la consecución de sus objetivos. Entonces, para que el proceso lector alcance parámetros de eficacia debe participar en actividades directamente relacionadas con la planificación, supervisión y evaluación del proceso (Stemberg, 1987; citado por Garner 1987a). Para lograr un proceso realmente eficaz, reflexivo, critico de lectura para aprender, se hace necesario que el lector asuma el control, la supervisión y la evaluación permanente de su propio proceso de comprensión. Es en este contexto donde se reconoce el papel de la metacognición.

Si lo cognitivo se relaciona con el conocimiento, lo metacognitivo se refiere al hecho de tener conciencia de tal conocimiento y de cómo se logra. En otras palabras, saber qué se sabe y no se sabe, así como también qué se conoce acerca del propio sistema cognitivo: capacidades y limitaciones y, por tanto, aquello que se tiene que hacer para llegar a saber. Mientras cognición se centra en procesos mentales como la percepción, la atención, la memoria y la comprensión, la metacognición se ocupa de la metapercepción, la metatención, la metamemoria y la metacomprensión (Bake y Brown 1984).

Los aspectos cognitivo y metacognitivo se encuentran ligados y en interacción. En el caso de la lectura llegan, incluso, a superponerse en algunas actividades como las relacionadas con los procesos de inferencia, la focalización de lo esencial y la elaboración de resúmenes, por ejemplo: La razón. Actividades como éstas suponen tanto el manejo de unos contenidos específicos como una supervisión permanente de lo logrado.

La claridad que tiene un lector en relación con el papel de los procesos mentales al momento de leer le permite saber qué necesita para conocer y comprender, qué está pasando mientras lee, cuál es su estado de comprensión del texto y darse cuenta durante el proceso si está comprendiendo o no lo que lee. En consecuencia, el lector puede regular su proceso de lectura y, en caso necesario, decidir acerca de la necesidad o conveniencia de usar otras alternativas más eficaces (López, 1997a). Se ha observado que las estrategias son específicas para cada texto y propósito de lectura; sin embargo, pueden, generalizarse a tipos de textos.

Flavell (1970, 1981 citado por Garner 1987b) y Garner (1987b), consideran los siguientes aspectos metacognitivos: el conocimiento metacognitivo incluye el conocimiento y las creencias acerca del conocimiento y de cómo se conoce en general; se encuentra almacenado en la memoria a largo plazo y se evoca como guía de las actividades cognitivas, las experiencias metacognitivas desarrolladas a partir de la experiencia, como lectores y se relaciona con los conocimientos propios y con la conciencia de los asuntos cognitivos y el uso estratégico procedimiento más adecuado para la consecución de las metas. Vale anotar, que Flavel resalta el papel de la experiencia y de los conocimientos metacognitivos. 
Finalmente, se ha de anotar cómo Garner (1987a) señala que la metacognición nace a partir de la doctrina piagetiana del desarrollo; se caracteriza porque su investigación utiliza, preferiblemente, entrevistas clínicas y porque los investigadores metacognitivos hablan de desarrollo del conocimiento, de conciencia y de acceso consciente. Por su parte, Fisher y Mandl (1984 y 1988) anotan que los postulados de Flavell pretenden enriquecer los conocimientos del sujeto para aumentar la eficacia mediante la instrucción de su funcionamiento cognitivo.

Estrategias metacognitivas de comprensión.

Mientras la cognición se centra en procesos mentales como la comprensión, la metacognición se ocupa de las estrategias metacognitivas de comprensión (Bake y Brown 1984). De manera particular, la "metacomprensión" permite al lector saber qué necesita para comprender, qué está pasando mientras lee, cuál es su estado de comprensión del texto y darse cuenta durante el proceso si está comprendiendo o no lo que lee. Además, el lector puede, en consecuencia, regular su proceso de lectura y, en caso necesario, decidir acerca de la necesidad o conveniencia de usar otras alternativas más eficaces (López, 1997a). Se ha observado que las estrategias, de la misma manera que las estrategias metacognitivas de comprensión, son específicas a cada texto y al propósito de lectura; sin embargo pueden generalizarse a tipos de textos.

Se entiende por estrategia la secuencia de actividades intencionales y deliberadas en las cuales se involucra conscientemente el individuo para lograr las metas que se ha propuesto. Weinstein y otros (1988) señalan determinadas características del aprendizaje estratégico: una estrategia...

- Está bajo el control del sujeto,

- Requiere mayor esfuerzo y recursos atencionales suficientes,

- Responde a una tarea específica,

- Permite determinar en qué hacer énfasis durante el proceso,

- Selecciona, de una serie de alternativas posibles, las actividades a desarrollar,

- Determina cuándo, dónde y cómo usar las actividades,

- Es un proceso flexible que se puede ir ajustando de acuerdo con el logro de las metas.

Diversas investigaciones han establecido diferencias entre lectores competentes y menos competentes en términos de estrategias. Según Garner (1987b), el 
buen lector se caracteriza por la forma como se relaciona e interactúa con el texto: tiene conciencia y control de sus actividades cognitivas y metacognitivas durante el proceso y, además, hace uso flexible de estrategias para ajustarlas a su propósito de lectura, a la tarea(s) específica(s) y al tipo de texto. Por su parte, Brown (1980), lo define como el individuo que tiene conciencia y control sobre sus actividades cognitivas mientras está involucrado en la lectura; en otras palabras, reconoce la necesidad de usar diferentes estrategias para diferentes tipos de textos. Identifica tres estrategias que usa un buen lector al leer:

- Establece un propósito para la lectura: activa conocimiento previo, genera hipótesis, predice, se pregunta y evalúa.

- Identifica y selecciona destrezas apropiadas de lectura.

- Controla su proceso de comprensión de la lectura; es decir, evalúa y determina cambios en el proceso (Baker, 1984).

León (1991), indica que los lectores menos competentes no son capaces de procesar ni de organizar la información nueva; esto les impide relacionarla con la que ya poseen; sus dificultades se deben, fundamentalmente, a la ineficacia en el momento de seleccionar e implementar estrategias adecuadas en la comprensión del texto. Para Garner (I987b), los lectores no eficientes, en general, no toman conciencia del proceso de lectura, no disponen de las estrategias adecuadas o no tienen flexibilidad ni habilidad para ajustar las que tienen hacia el logro de un proceso óptimo.

De esta forma, esta investigación logró identificar la claridad que tiene el lector frente a su accionar cognitivo durante el proceso de lectura. En tal sentido, se ha insistido en que la lectura consiste en una compleja actividad constituida por diversos procesos cognitivos; en consecuencia, puede verse afectada la comprensión si se afecta cualquiera de tales procesos.

De acuerdo con lo planteado hasta ahora, para lograr un proceso eficaz, reflexivo, critico de lectura para aprender, se hace necesario que el lector asuma el control, la supervisión y la evaluación permanente de su propio proceso de comprensión. Aquí entran en juego las estrategias metacognitivas de comprensión. El estudiante universitario, lector adulto, si asume la lectura como proceso para aprender, si hace uso de estrategias que le permitan el logro de conocimiento, de saber, reconocerá de la necesidad de supervisar y regular permanentemente su propio proceso. Por su parte, el docente, además de asignar lecturas específicas, propondrá tareas que hagan evidente el uso de tales estrategias para acompañar procesos eficaces.

Con el fin de atender la consecución de los dos objetivos básicos de la metacomprensión (la toma de conciencia de la lectura como proceso estratégico 
y de sus implicaciones en el proceso de comprensión y el autocontrol y la autorregulación de todo el proceso), distintos autores han propuesto tres etapas del desarrollo de la metacomprensión:

\section{Planificación.}

Precisar los objetivos y las metas de lectura, los conocimientos en relación con el tema, el plan de acción y las estrategias a usar:

1. Tener presente el propósito de la lectura.

2. Planificar el proceso de lectura para decidir qué hacer para lograr el propósito: qué estrategias usar, qué procedimiento seguir.

3. Enfocar selectivamente la atención en lo esencial del texto.

\section{Supervisión.}

Comprobar si se está llevando a cabo lo planificado; verificar la eficacia y la adecuación de las estrategias a los objetivos; si se encuentran dificultades determinar a qué pueden ser debidas y cómo resolverlas:

1. Tomar notas en relación con la información relevante.

2. Elaborar el resumen de los contenidos.

3. Tomar decisiones acerca de la necesidad o conveniencia de hacer los correctivos pertinentes.

Este último punto en particular supone: tomar conciencia de la posibilidad de tener fallas en comprensión, identificar la fuente de las fallas y decidir qué hacer.

\section{Evaluación.}

Tanto los procesos desarrollados durante la lectura, como de los resultados obtenidos. Supone, entonces, no sólo constatar el nivel de comprensión que se va alcanzando, sino, además, evaluar durante todo el proceso, las distintas operaciones que constituyen la actividad lectora:

Formularse preguntas acerca de lo que se va leyendo, para determinar si está comprendiendo.

Evaluar permanentemente el proceso en su desarrollo, así como sus resultados y comprobar la eficacia de las estrategias y del procedimiento utilizado en la consecución de los objetivos planteados.

A manera de conclusión, la lectura implica organizar la experiencia y establecer relaciones entre las ideas tomads de diferentes fuentes. La lectura, 
en "el proceso de aprender a aprender", facilita el logro y el desarrollo de procesos cognitivos y metacognitivos en aras de un desempeño académico que promueva la construcción del conocimiento y de desarrollo científico para contribuir en la transformación de la sociedad.

Los avances académicos propios de la educación superior demandan que la comprensión de lectura se ubique en contextos de uso, pues aprender a partir de los textos se constituye aquí en un acto ineludible. Explicitar y sistematizar las estrategias utilizadas para la comprensión de lectura abre vías para que los lectores puedan reconocerlas y desarrollarlas de manera pertinente en los niveles de educación superior. Por consiguiente, el énfasis recae en los distintos mecanismos que se ponen en juego en la lectura comprensiva.

En Educación Superior, y especialmente en la educación virtual, se hace necesario que los estudiantes se acerquen de manera significativa y continua a diversas producciones textuales especialmente a las de carácter académico. Las condiciones vigentes en los procesos de formación universitaria requieren cualidades frente a determinadas exigencias en la comprensión de textos. El siglo XXI, caracterizado por el dinámico y veloz flujo de información, demanda que el sistema educativo escolarizado camine a la par. Tanta información ofrecida por diversos medios y modalidades exige desarrollar de estrategias adecuadas para el procesamiento y la comprensión de textos.

\section{Referencias bibliográficas}

Adams M. J. (1980). "Failures to comprehend and levels of processing in reading”. En: R.J Spiro. Bruce B.C. y Brewer W.F.. Theoretical Issues in Reading Comprehension, Hillsdale. New Jersey: Lawrence Erlbaurn Assoc.

Anderson. T. M. Y Armbruster, B. (1984). “Studying”. En P. Pearson (ed.), Handbook of Reading Research. N. York. Longman.

Alliende, F. (1982). "La comprensión de la lectura y su desafio". En: Lectura y vida, 3. I. de Beaugrande, R (1980). Text, Discourse and Process, New Jersey: Ablex Pub. Corp. de Beaugrande R. y DresslerW. 1983 Introduction to Text Linguistics. London: Longman.

Bransford J.D. (1985). "Schema activation and schema adquisition”. En: R Singer y R. B. Rudell (ed), Theoretical Models and Processes of Reading, Newark: International Reading Association.

Bransford J.D. y Johnson M.K. (1990). "Consideraciones sobre algunos problemas de la comprension”. F. Valle y otros (ed). Lecturas de Sociolinguística. Alianza. Madrid 


\section{Sandra Acevedo Zapata y Lilia Cañón Flórez}

Análisis sobre los procesos cognitivos implícitos en la comprensión de textos. Artículo producto de la investigación.

Brown, A L. (1980). “Metacognitive Development and Reading” En R. J. Spiro, B. C: Bruce y F. Brewer (eds.), Theoretical issues in Reading Comprehension. Hillsdale, Nueva Jersey; Earlbaum.

Coulthard, M. (1977). An Introduction to Discourse Analysis. Inglaterra Longman. Fischer P. M. y Mandi H. 1984 "Learner, text variables, and the control of the text comprehension and recall”. H. Maudl y otros (ed), Learning and Comprehension of Text. Lawrence Erlbaurn Assoc . N.J. 213-254.

Garner, R (1986). "Children's knowledge of structural properties of expository texts". Journal of educational Psychology”. 78: 6, 411-416.

Garner, R (1987) a. "Strategies for reading and studying expository texts". Educational Psychologist, $22: 3-4,2997312$.

Garner, R (1987) b. Metacognition and Reading Comprehension. Ablex Publishing Corporation. N.J., USA

Garnham A. y Oakhill J. (1992). "Discourse and text processing from a mental models perspective”. Language and Co.gnitive Processes, 7 : 3,4, 193-204.

González Marques J. (1991). “Las inferencias durante el proceso lector”. A Puente (comp)

León J. A. (1991) a. “La mejora de la comprensión de lectura” En infancia y aprendizaje N 56.

López, G. S. y Conover, N. (1980). "Determinación de necesidades en la enseñanzaAprendizaje de idiomas Extranjeros”. Informe de Investigaci6n. En Revista Lenguaje 11. Departamento de Idiomas, UNIVALLE.

López, G. S (1997) a. "La metacomprensión y la lectura”. En M. Martínez (Comp.) Los Procesos de la Lectura y la Escritura, Cali: Universidad del Valle.

Lopez, G. S (1997) b. "Los Esquemas como facilitadores de la comprensi6n y aprendizaje de textos”. En Revista Lenguaje 26. Cali: Edit. Facultad de Humanidades. Universidad del Valle.

Lesgold M. A. y Perfetti Ch. A. (ed) Hillsdale. N.J. (1981). Interactive Processes in Reading, Erlbaum.

Martínez M. C. (1994). Análisis del Discurso. Cali: Centro Edit. Universidad del Valle.

Martínez M. C. (1997). EI desarrollo de estrategias discursivas a nivel universitario”. En M. C. Martínez (comp.) Los Procesos de la Lectura y la Escritura. Cali: Edit. Universidad del Valle. 
Newell. A (1980). “Inteligencia Artificial y Concepto de la Mente”. Valencia: Revista Teorema.

Orasanu J. (ed) (1986). Reading Comprehension: from Research to Practice, Erlbaum Hillsdale. N. J.

Orasanu J (ed.) Reading Comprehension: from Research to Practice, Erlbaum Hillsdale, N.J. Rumelhart D. E. (1977) a. "Toward an interactive model of reading”. S. Dornic (ed), Atention and Performance, vol. VI. Academic Press, NY

Rumelhart, D. E. (1977) b. “Understanding and summarizing brief stories”. D. LaBerge y S. J. Samuels, Basic Processes in Reading, Hillsdale, Erlbaum. N.J.

Rumelhart D. E. (1980) a. "Schemata: the building blocks of cognition". R. J. Spiro y otros (ed) Theoretical issues in Reading Comprehension, Hillsdale, N.J.

Rumelhart, D. E. (1984). “Understanding understanding”. J. Flood (ed), Understanding Reading Comprehension

Sinclair, J. M. Y Coulthard R. (1975). An Introduction to Discourse Analysis. Inglaterra. Longman.

Singer, H y R. B. Rudell (ed) (1994). Theoretical Models and Processes of Reading, IRA Newark, Delaware.

Smith F. (1971). Reading, Cambridge University Press, Cambridge.

Smith F. (1990). Para darle Sentido a la Lectura, Visor, Madrid

Spiro, R. J. (1979). Ethiology of Reading Comprehension Style. Urbana, U. of Illinois.

Spiro, R. J (1980). “Constructive Pocesses in Prose Recall”. En R. J. Spiro, B. Bruce y W. Brewer (eds.): Theoretical issues in Reading Comprehension: Prespectives from Cognitive Psichology. Linguistics, Artificial Intelligence, and Education. Hillsdale, N. J.: Erlbaum.

Tierney, R. J. y Pearson P. D. (1985). "Learning to learn from text". H. Singer y R. B. Rudell (ed) Theoretical Models and Processes of Reading, LRA, Newark Delaware.

Widdowson H. (1978). Teaching language as Communication, Oxford: O.U.P.

Widdowson H. (1984). "Reading and Communication". Explorations in Applied Linguistics. Oxford: O.U.P. 